\title{
Introducing a Mobility Access Category for IEEE 802.11e
}

\section{Mohamed Aboulwafa, Nourddine Enneya}

\begin{abstract}
With traffic increase in a wireless network beyond its capacity and as the number of connected devices continue to grow, the quality of service (QoS) degrades. In this paper we study the impact of mobility on throughput in the case of an infrastructure wireless network using IEEE 802.11 Wi-Fi standard. Since we found in the literature that the mobility of stations can have an impact on the quality of service, we try to remedy to this by implementing a new access category reserved for mobile stations. First we compare the throughput between static and mobile nodes, both connected to a QoS station. Then we propose our new model that consists of adding a new access category used by mobile nodes regardless of their traffic category. The study was made by simulating different scenarios using Network Simulator-3 (NS-3). We found that the throughput may vary depending on the simulation scenario. The simulation results show that with the proposed solution the mobile nodes can have a better throughput.
\end{abstract}

Keywords: 802.11e, EDCA, QoS, Mobility, MAC

\section{INTRODUCTION}

The IEEE 802.11 protocol has become the most dominant and is vastly used in Wireless Local Area Networks (WLANs) [1]. IEEE 802.11 WLANs are easily deployed with low cost in zones like airports, office, hotels and residence homes. With an increasing popularity of multimedia applications (VoIP and streaming) that requires a guaranteed QoS. QoS stations (STAs) allow a better use of the network by providing prioritized QoS services which classify all the traffic to multiple Access Categories (ACs). The protocol includes the 802.11e standard that proposes a new function for the MAC layer, known as the Hybrid Coordination Function (HCF). This function uses a contention channel access method based on EDCA. Enhanced Distributed Channel Access (EDCA) is designed to provide prioritized QoS and improve the Distributed Coordination Function (DCF) belonging to the original 802.11 standard. It is important to study the impact of mobility on different protocols and scenarios as the performance of different network protocols can be affected in different ways, in this paper we chose to study the impact of mobility on throughput in IEEE 802.11n protocol and we choose random walk mobility model as it is close to a normal usage of wireless devices around the office or in a public space.

Revised Manuscript Received on March 30, 2020.

* Correspondence Author

Mohamed Aboulwafa, PhD, Laboratory of Informatics systems and optimization at the University of Ibn Tofail (Kenitra- Morocco)

Nourddine Enneya, Associate Professor; elected as head of the the Laboratory of Informatics, Systems and Optimization, within the aforementioned university

(C) The Authors. Published by Blue Eyes Intelligence Engineering and Sciences Publication (BEIESP). This is an open access article under the CC BY-NC-ND license (http://creativecommons.org/licenses/by-nc-nd/4.0/)
The aim of this study is to compare the throughput between static and mobile nodes in the case of classic EDCA parameters and in the case of our new access category. This paper is organized as follows; Section 2 describes basic theory of WLAN IEEE 802.11e and EDCA MAC layer, Section 3 describes some related work. In Section 4, we enunciate our proposed model. In section 5 , we evaluate the impact of mobility on throughput via simulations and present the obtained results. Finally, Section 6 concludes the paper.

\section{IEEE 802.11E EDCA OVERVIEW}

According to IEEE standard 802.11 the mobility of wireless nodes should be handled within the MAC sub-layer, as the IEEE Std 802.11 is required to appear to higher layers [logical link control (LLC)] as a wired IEEE 802 LAN [1]. The fundamental access method of the IEEE 802.11 MAC is a Distributed Coordination Function (DCF) known as carrier sense multiple access with collision avoidance (CSMA/CA). It is required that the Distributed Coordination Function must be implemented in all stations (STAs). For a STA to transmit, it has to sense the medium to check if it currently used by another transmitting station. If the medium is determined to be free, the transmission may proceed.

In order to achieve traffic differentiation, 802.11e Enhanced Distributed Channel Access (EDCA) assigns higher priority Access Categories (ACs) with smaller minimum Contention Window (CWmin), maximum Contention Window (CWmax), and Arbitration Inter-Frame Space (AIFS) to influence the successful transmission probability (statistically) in favor of high-priority ACs, the probability of gaining access to the medium gets higher if the parameter values (AIFS, CWmin/ CWmax) are smaller. Each AC has its own queue within a station that behaves like an individual virtual station, the access category contends for access to the medium and independently starts its backoff procedure after detecting the channel being idle for at least an AIFS period. The backoff procedure of each AC is the same as that of DCF. Fig. 1 shows the timing diagram for the EDCA. For the contention process to transmit a frame belonging to an AC, EDCA uses AIFS[AC], CWmin[AC], and CWmax[AC]. The smaller AIFS and CWmin for an access category, the shorter the channel access delay for its corresponding priority, which allows a given traffic condition to have more capacity share. These parameters are used in order to differentiate the access to the channel among the different traffic priorities [2]. Each frame from the higher layer arrives at the MAC along with a specific priority value called User Priority (UP), originally there are eight different user priorities. 
Then, each data frame carries its priority value in the MAC frame header, as every 802.11e station shall implement four access categories (ACs) which are shown (see Figure 2). The access categories are derived from the user priorities as shown in Table 1. [1]

When a collision occurs among different ACs within the same station, the lower priority AC suffers from a virtual collision, similar to a real one outside the station, while the access category with higher priority is granted the opportunity to transmit, Fig.2 [3] shows the four access categories inside a QoS station.

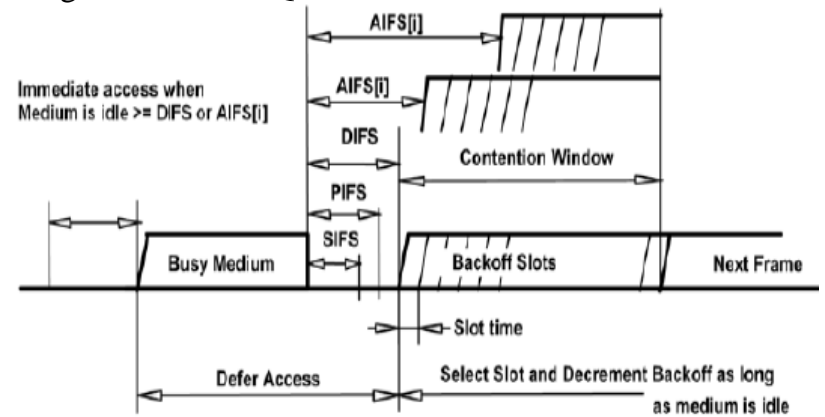

Fig. 1 802.11e EDCA Channel Access

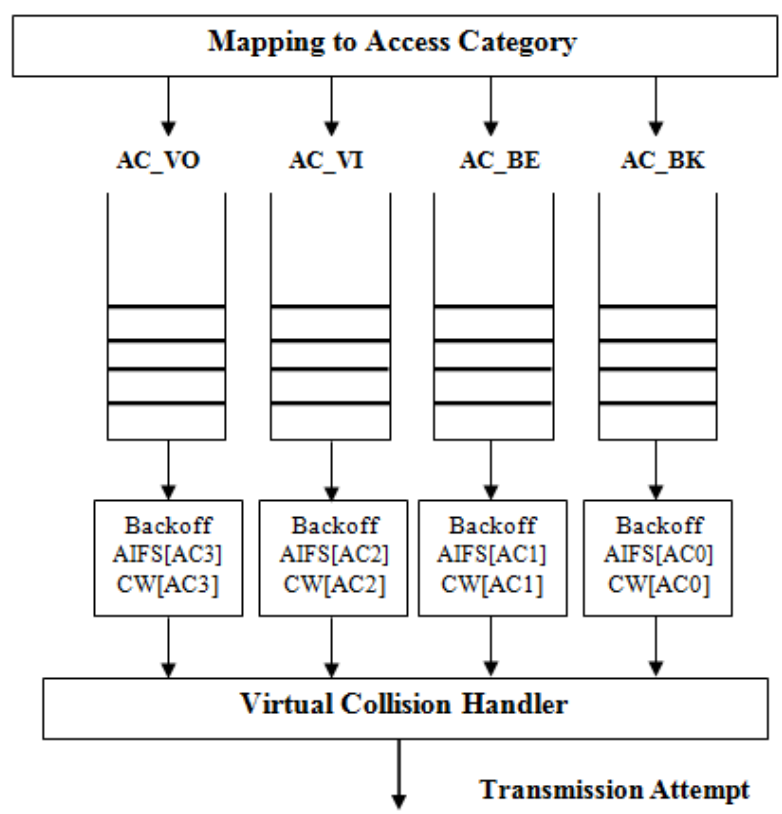

Fig. 2 Four Access Categories of 802.11e EDCA

\section{RELATED WORK}

Studying the literature for the subject comes down to two main parts, the first part are the studies on the impact of mobility on quality of service, and the second is the optimization based on the modification of EDCA parameters depending on the context and on the type of packets that needs to be sent.

\section{A. Impact of mobility on QoS}

Lenders et al. made a real life experiment where they found that for links with a long lifetime, mobility is the most dominant cause of link failure, whereas for the breakage of links with short lifetimes causes unrelated to mobility are responsible[5].

Table - I : UP-to-AC mappings

\begin{tabular}{|c|c|c|c|c|}
\hline Priority & $\begin{array}{c}\text { UP } \\
\text { (Same as } \\
\text { s02.1D user } \\
\text { priority) }\end{array}$ & $\begin{array}{c}802.1 \mathrm{D} \\
\text { designation }\end{array}$ & AC & $\begin{array}{c}\text { Designation } \\
\text { (informative) }\end{array}$ \\
\hline \multirow[t]{6}{*}{ Lowest } & 1 & BK & AC_BK & Background \\
\hline & 2 & - & AC_BK & Background \\
\hline & 0 & $\mathrm{BE}$ & AC_BE & Best Effort \\
\hline & 3 & $\mathrm{EE}$ & AC_BE & Best Effort \\
\hline & 4 & $\mathrm{CL}$ & AC_VI & Video \\
\hline & 5 & vI & AC_VI & Video \\
\hline$\nabla$ & 6 & vo & AC_vo & Voice \\
\hline Highest & 7 & $\mathrm{NC}$ & AC_VO & Voice \\
\hline
\end{tabular}

Grossglauser et al. have showed that the throughput scaling changes completely at the time of stations movement [6]. Gowrishankar et al. tested the impact of different random mobility models (Random Waypoint, Random Walk with Reflections and Random Walk with Wrapping) on the performance of AODV routing protocol, the experiment results show that the performance varies according to the mobility model [7].

Zogkou et al. made simulations where they show that the velocity of the nodes impacts the number of packets that are successfully received by the destination node. Since nodes mobility results in more connection loss, the Packet Delivery Ratio among nodes slightly decreases. Moreover, it takes more time to deliver packets from source nodes to destination nodes and therefore the average delay increases [8].

Banchs et al. concluded that a new concept for admission control is required under mobility conditions, so that it can constantly monitor the channel conditions and reevaluates admission control decisions [9].

Dridi et al. found that a low mobility offers the best throughput for real-time applications and videoconferencing, and a high mobility can be satisfactory for email but not satisfactory for other types of traffic [10].

\section{B. EDCA parameters based optimization}

Pérez et al. made a study about the impact of EDCA parameters the Wi-Fi QoS, they found that the default parameters are not the ones that provide the best performance, and that AIFSN parameter has more important impact for ensuring QoS than other parameters [11].

Koukoutsidis et al. studied the joint settings of the EDCA parameters in order to maximize performance, their findings show that drastic service differentiation can undermine the capacity of a WLAN, and they reveal different optimal settings for different load conditions [12].

Flaithearta et al. described how they used synchronized time to provide QoS improvements especially to VoIP through 802.11e parameter optimization [13].

\section{PROPOSED APPROACH}

A fundamental characteristic of mobile wireless networks is the variation of the channel strength of the underlying communication links. Different simulation results show that the mobility of a given station have an impact on its throughput. 
We try to remedy to this by adding a new access category to the EDCA existing four access categories, we call the new AC "Access Category Mobile” (AC_MO).

In our proposed model the new AC_MO will have the highest priority, the traffic directed toward a mobile station will be directed through AC_MO. To prevent the mobile nodes of monopolizing the medium AC_MO will be given a smaller TXOP value.

The access method to the medium will remain the same as we described before for the 802.11e EDCA, except that in this model the packets will be directed to five queues instead of the original four (Fig.3), if the station is detected as mobile, whatever access category the traffic belongs to, it will be diverted to the access category Mobile, following the algorithm below:

\section{Algorithm of allocation of AC_MO}

1. for $\mathrm{i}=0$ until $\mathrm{i}=$ number of nodes

2. if node(i) is mobile then

3. node(i). AC $\leftarrow$ AC_MO

4. else

5. node(i) gets AC according to its traffic category

6. endif

7. increment $\mathrm{i}$

8. endfor

\section{SIMULATION SETUP AND RESULTS DISCUSSION}

In the simulations of standard EDCA model, we found that there is a degradation of throughput that affects the mobile stations. After testing our proposed model we found that there is significant improvement in throughput.

\section{A. Simulation Setup}

For the simulations we use NS-3 simulator to evaluate the impact of mobility on throughput in IEEE 802.11, we choose 802.11n as the PHY layer. The mobility of the nodes is according to the random walk mobility model, where nodes move with random speed in a direction chosen randomly between $0^{\circ}$ and $360^{\circ}$. To test our model we relied on two simulation sets:

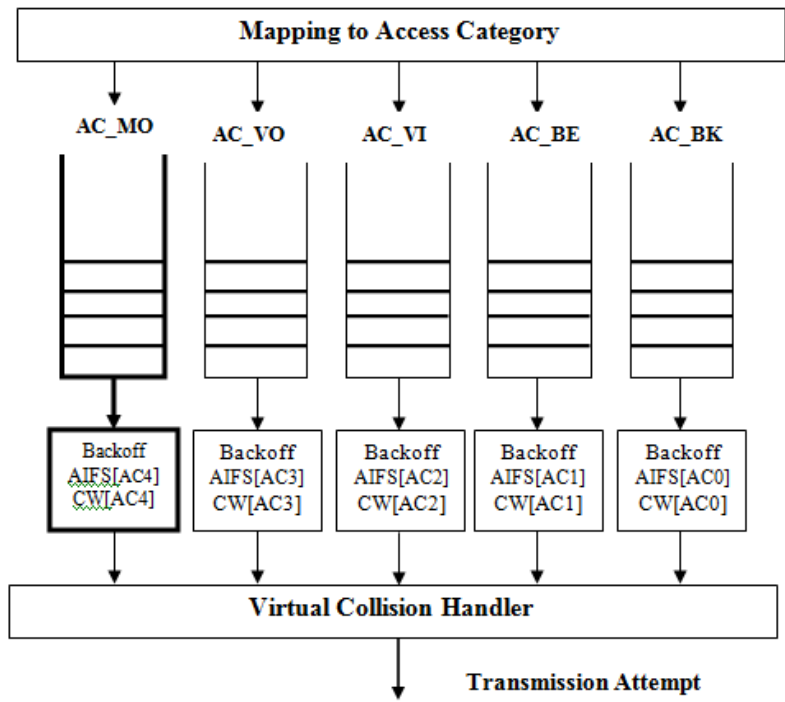

Fig. 3 New Mobile Access Category

\section{Simulation set 1}

To compare the throughput of static and mobile stations we have considered two scenarios, namely scenario 1 and scenario 2. In each scenario 10 stations (STAs) are connected to the same access point (AP).

Scenario 1 consists of all the STAs given fixed positions and connected to the access point with an access category of type Voice (AC_VO).

Scenario 2 consists of all STAs being mobile according to the random walk mobility model, and connected to the access point with an access category of type Voice (AC_VO).

Table - II : Simulation set 1

\begin{tabular}{|c|c|c|c|}
\hline & Access Point & Stations & \multicolumn{1}{c|}{ Access Category } \\
\hline Scenario 1 & 1 & 10 Fixed & AC_VO \\
\hline Scenario 2 & 1 & 10 Mobile & AC_VO \\
\hline
\end{tabular}

We compare the throughput for each station by simulating both scenarios, the results represent the mean value of 10 simulations for the same scenario. The results are shown in Figure 4.

\section{Simulation set 2}

To compare the throughput of stations connected with different Access Categories, we have a total of 20 stations connected to the same access point (AP), all the stations are mobile using the random walk mobility model, 10 stations have static positions and are connected to the AP using the access category of type voice (AC_VO) and the remaining stations are mobile and connected to the AP using the new access category of type mobile (AC_MO).

\section{Table - III : Simulation set 2}

\begin{tabular}{|c|c|c|}
\hline Access Point & Stations & Access Category \\
\hline \multirow{2}{*}{1} & 10 Fixe & AC_VO \\
\cline { 2 - 3 } & 10 Mobile & AC_MO \\
\hline
\end{tabular}

We compare the throughputs between the two access categories, the results represent the mean value of 10 simulations and are shown in Figure 5.

\section{B. Quality of Service Metrics}

Quality of Service (QoS) reflects the service availability and transmission quality, it is defined as the measure of performance for a transmission system. Service availability is a crucial element of QoS. It should be noted that the network infrastructure must be highly available before QoS can be successfully implemented. [4]The network transmission quality is determined by:

Throughput: refers to the quantity of data that can be transferred from one station to another in a given amount of time (in this paper we use Mbits/s).

Delay: ( or latency) called also end-to-end delay is the amount of time it takes for a packet to go from the sending end point the receiving end point after being transmitted. This time period can be divided to fixed and variable network delay:

- Fixed network delay-Includes encoding and decoding time (for voice and video), and the amount of time required for the signal to traverse the media en route to its destination. 
- Variable network delay-Generally refers to network conditions that can affect the overall time required for transit, such as queuing and congestion.

Jitter : (or delay-variance) is the difference in the end-toend delay between packets.

Loss : (or packet loss) is expressed as the percentage of packets that were dropped. Loss is a comparative measure of packets successfully transmitted and received to the total number that were transmitted.

\section{Results and discussion}

The results of simulation 1 show that while using the same access category (AC_VO), the difference in throughput between the static and mobile stations is considerable (in the order of $10 \mathrm{Mbits} / \mathrm{s}$ ) which lead us to think that mobility of a node can have an impact on its throughput.

Based on the results of simulation 1, we decided to add a new access category that will be dedicated for mobile nodes. To test the impact of the new AC on mobile nodes, we used the setup described in simulation set 2 in which 10 stations are connected to the AP with $\mathrm{AC} \_\mathrm{VO}$ and the other 10 STAs connected to the AP with AC_MO. The results show that difference in throughput between the two sets of nodes was reduced and the mobility does not impact the throughput as much as it did before we introduced the mobile access category (AC_MO). The difference in throughput between the two simulations is due to the number of stations connected to the access point, in simulation 1 only 10 stations are connected at a time, but in simulation 2 since we want to compare the throughputs of mobile and static STAs and in order to have a better comparison 20 stations are connected to the access point at the same time.

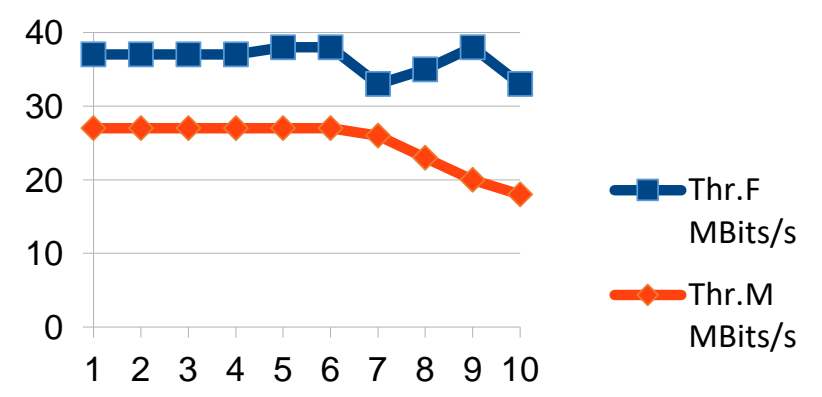

Fig. 4 Throughput of fixed and mobile nodes in Mbit/s.

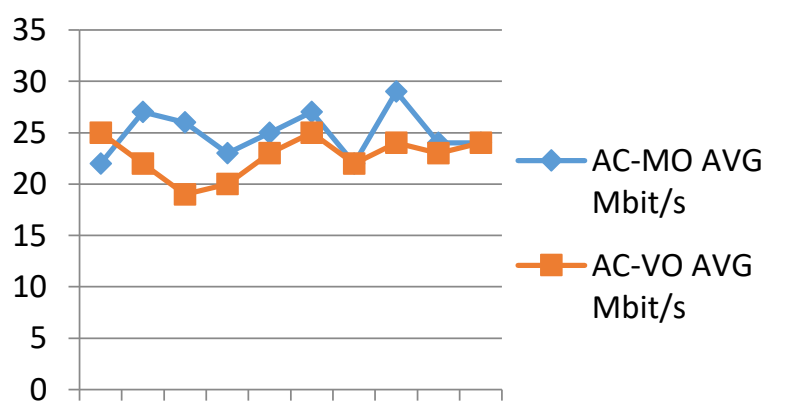

$\begin{array}{llllllllll}1 & 2 & 3 & 4 & 5 & 6 & 7 & 8 & 9 & 10\end{array}$

\section{CONCLUSION}

In this paper we have evaluated the impact of mobility on throughput through simulations, we observed that the mobile stations had a lower throughput compared to the static stations, and we tested via simulation our proposed model which consists of adding a new access category and allocating it only for mobile nodes regardless of their original traffic category. The tests show that the difference in throughput between mobile and static stations was reduced considerably in the case of mobile access category. The purpose of this study was to test our theory about the impact of mobility and if the proposed model could help with throughput fairness, it turns out that in the case where there are both mobile and static stations connected to the same access point, the throughput of the mobile stations becomes low compared to the static ones, and with the modifications made to the MAC layer it becomes possible to achieve fairness between the different stations.

\section{REFERENCES}

1. IEEE Std 802.11, "Part 11: Wireless LAN Medium Access Control (MAC) and Physical Layer (PHY) Specifications", March 2012

2. Sari, Riri Fitri, Yan Maraden, and Kamal Djunaedi. "Performance Evaluation of IEEE 802.11 e EDCA based on Variable Priority Parameters." Proceedings of Quality in Research (QIR) Conference. 2007.

3. Cai, Lin X., et al. "Supporting voice and video applications over IEEE 802.11 n WLANs." Wireless Networks 15.4 (2009): 443-454.

4. Mohapatra, Prasant, Jian Li, and Chao Gui. "QoS in mobile ad hoc networks." IEEE Wireless Communications 10.3 (2003): 44-53.

5. Lenders, V., Wagner, J., Heimlicher, S., May, M., \& Plattner, B. (2008). An empirical study of the impact of mobility on link failures in an 802.11 ad hoc network. IEEE Wireless Communications, 15(6), 16-21.

6. Grossglauser, Matthias, and David NC Tse. "Mobility increases the capacity of ad hoc wireless networks." IEEE/ACM transactions on networking 10.4 (2002): 477-486.

7. Gowrishankar, S., T. G. Basavaraju, and Subir Kumar Sarkar. "Effect of random mobility models pattern in mobile ad hoc networks." International Journal of Computer Science and Network Security 7.6 (2007): 160-164

8. Zogkou, Maria, et al. "EDCA mechanism and mobility support evaluation in IEEE 802.11 s WMNs." 2014 6th International Congress on Ultra Modern Telecommunications and Control Systems and Workshops (ICUMT). IEEE, 2014.

9. Banchs, Albert, et al. "Applications and challenges of the 802.11 e EDCA mechanism: an experimental study." IEEE network 19.4 (2005): 52-58.

10. Dridi, Khaled, et al. "IEEE 802.11 e-EDCF evaluation through MAClayer metrics over QoS-aware mobility constraints." Proceedings of the 7th International Conference on Advances in Mobile Computing and Multimedia. 2009.

11. Pérez, Santiago, et al. Analysis of Impact in the Wi-Fi QoS of the EDCA Parameters. No. ART-2015-95158. 2015.

12. Koukoutsidis, Ioannis, and Vasilios A. Siris. "802.11 e EDCA protocol parameterization: A modeling and optimization study." 2007 IEEE International Symposium on a World of Wireless, Mobile and Multimedia Networks. IEEE, 2007.

13. Flaithearta, Padraig O., Hugh Melvin, and Michael Schukat. "Optimising QoS of VoIP over wireless LANs via synchronized time." 2013 IEEE 14th International Symposium on" A World of Wireless, Mobile and Multimedia Networks"(WoWMoM). IEEE, 2013.
Fig. 5 Throughput of nodes connected with AC_VO and AC_MO in Mbits/s. 


\section{AUTHORS PROFILE}

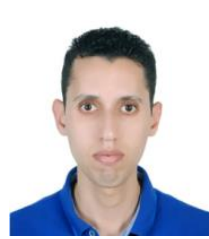

Mohamed Aboulwafa holds a master's degree in Mathematics and Informatics form the National School of Applied Sciences (ENSA- Tangier,Morocco) since 2013, and is currently pursuing a PhD at Laboratory of Informatics systems and optimization at the University of Ibn Tofail (Kenitra- Morocco). His research interests are mainly focused on Wireless Networks, Computer Engineering and the Enhancement of the Quality of Service in wireless local area networks.

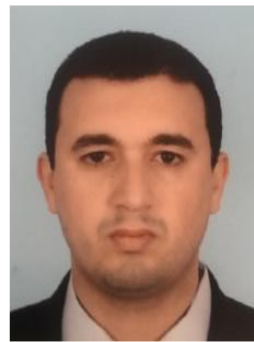

Nourddine Enneya earned his $\mathrm{PhD}$ in Computer Science from the University of Mohammed V (Rabat, Morocco) in 2009, whereby he was appointed as Assistant Professor in the university of Ibn-Tofail (Kenitra, Morocco). As of 2013, he was promoted to Associate Professor; and elected as head of the the Laboratory of Informatics, Systems and Optimization, within the aforementioned university. His fields of expertise include, among others: QoS, QoE, CE and Wireless networks Security Protocols (with and without infrastructure). 